\section{Drug-induced hepatotoxicity studied in a 3D, in vitro model of the liver}

\author{
Giuseppe Guagliano, ${ }^{1}$ \\ Manuela Legnardim, ${ }^{1}$ Cristina Volpini, ${ }^{2}$ \\ Nora Bloise, 2 Silvia Dotti, ${ }^{3}$ Riccardo \\ Villa, ${ }^{3}$ Livia Visai, 2,4 Paola Petrini1 \\ 1Department of Chemistry, Materials \\ and Chemical Engineering "Giulio \\ Natta", Politecnico di Milano, Milan; \\ ${ }^{2}$ Molecular Medicine Department \\ (DMM), Center for Health Technologies \\ (CHT), UdR INSTM, University of \\ Pavia, Pavia; ${ }^{3}$ National Reference \\ Center for Alternative Methods, Welfare \\ and Care of Laboratory Animals, \\ Istituto Zooprofilattico Sperimentale \\ della Lomabardia ed Emilia Romagna, \\ Brescia; ${ }^{4}$ Medicina Clinica-Specialistica, \\ UOR5 Laboratorio di Nanotecnologie, \\ ICS Maugeri, IRCCS Pavia, Italy.
}

\begin{abstract}
Drug development is a high failure rate process; too many drugs fail during clinical trials because of severe hepatotoxicity. This situation can be significantly improved by redesigning preclinical trials, and privileging the use of high-throughput in vitro models, by combining cell-based in vitro models and material-based models.
\end{abstract}

\section{Introduction}

In the context of drug development, liver plays a crucial role, and it is primarily involved in drug metabolism. However, the direct exposition to the active principles and their metabolites may damage hepatocytes, triggering a macrophages-mediated cascade, culminating with the differentiation of hepatic stellate cells in myofibroblast, and producing fibrous matrix. The threshold beyond which a certain molecule become hepatotoxic varies within individuals subpopulations, since it is strictly dependant by the pharmacokinetics, ${ }^{1}$ and therefore it is difficult to be uniquely predicted. This work will present the first developmental steps of a tailorable and standardizable in vitro 3Dmodel of the liver, to assess drugs hepatotoxicity.

\section{Materials and Methods}

ECM was obtained from decellularized porcine liver, by a combination of different methods. ${ }^{2}$ The decellularization buffer was injected in multiple sites of $0.5 \mathrm{~cm}$ cubes of liver and then used to incubate the samples while under orbital stirring up to 7 days. Lyophilized cubes were grinded after freezing in liquid $\mathrm{N}_{2}$. ECM powder $(1.4 \% \mathrm{w} / \mathrm{v})$ was added in an Alginate (ALG) solution $(3.5 \% \mathrm{w} / \mathrm{v})$ in complete medium. The hydrogel was characterized by rheological testing and the stability in medium was evaluated up to 14 days. For cell loaded hydrogels, HepG2 cells were suspended in the ALG-ECM suspension $\left(2 \times 10^{6}\right.$ cell $\left./ \mathrm{mL}\right)$ prior crosslinking. ${ }^{3}$ MTT test and confocal microscopy, with live/dead kit were employed to evaluate viability and spatial distribution.

\section{Results and Discussion}

The produced hydrogel shows rheological characteristics reproducing the ones of the liver tissue and is stable up to 12 days. Additionally, further rheological analyses demonstrated the suitability of the produced hydrogel to be processed via $3 \mathrm{D}$ bioprinting.

Both qualitative (CLSM) and quantitative (MTT assay) viability analyses revealed that the number of cells within the hydrogel have increased through time. In particular, it has been demonstrated the feasibility of maintaining viable cells in culture up to 8 days. Additionally, CLSM analyses allowed to observe the formation of threedimensional cellular clusters, similar to the ones in which hepatocytes are organized in vivo. 4

The model is now being employed for the study of hepatoxicity to the administration of various drugs. Acetaminophen, whose hepatotoxic effects are known and well described, has been exploited to benchmark the model, and to calibrate it for the study of other substances (i.e., midazolam, chlordiazepoxide).

\section{Conclusions}

This study demonstrated the possibility to realize a drug-induced hepatotoxicity model by synergically combining the chemical features of liver-derived ECM with the structural characteristic of an alginate hydrogel, mimicking the in vivo liver microenvironment. The presence of ECM positively impacted cell viability.
Correspondence: Giuseppe Guagliano, Department of Chemistry, Materials and Chemical Engineering "Giulio Natta", Politecnico di Milano, Milan, Italy.

E-mail: giuseppe.guagliano@polimi.it

Key words: Hepatotoxicity; in vitro models; 3D models; alternative models.

Acknowledgments: Research project funded by the Italian Ministry of Health (Prot. n. 0025345 del 04/12/2020).

Disclosures: The authors declare that the research was conducted in the absence of any commercial or financial relationships that could be construed as a potential conflict of interest.

Conference presentation: This paper was presented at the Third Centro 3R Annual Meeting - L'era delle 3R: modelli in silico, in vitro e in vivo per promuovere la ricerca traslazionale 30 September - 1 October 2021, Evento online organizzato dal Politecnico di Torino.

Received for publication: 9 July 2021. Accepted for publication: 7 September 2021.

This work is licensed under a Creative Commons Attribution NonCommercial 4.0 License (CC BY-NC 4.0).

(C) Copyright: the Author(s), 2021

Licensee PAGEPress, Italy

Biomedical Science and Engineering 2021; 4(s1):172 doi:10.4081/bse.2021.172

\section{References}

1. Rodighiero V. Effects of Liver Disease on Pharmacokinetics An Update. Princ Clin Pharmacol 2012;37:73-87.

2. Mazza G, Al-Akkad W, Telese A, et al. Rapid production of human liver scaffolds for functional tissue engineering by high shear stress oscillation-decellularization. Sci Rep 2017;7:5534.

3. Lan SF, Safiejko-Mroczka B, Starly B. Long-term cultivation of HepG2 liver cells encapsulated in alginate hydrogels: a study of cell viability, morphology and drug metabolism. Toxicol In Vitro 2010;24:1314-23.

4. Godoy P, Hewitt NJ, Albrecht U, et al. Recent advances in $2 \mathrm{D}$ and $3 \mathrm{D}$ in vitro systems using primary hepatocytes, alternative hepatocyte sources and nonparenchymal liver cells and their use in investigating mechanisms of hepatotoxicity, cell signaling and ADME. Arch Toxicol 2013;87:1315-530. 